\title{
ASSOCIAÇÃO ENTRE DEFICIÊNCIA DE VITAMINA A E SITUAÇÃO SOCIODEMOGRÁFICA DE MÄES E RECÉM-NASCIDOS
}

\author{
Rejane Andréa Ramalho*, Hernando Flores, Elizabeth Accioly, Cláudia Saunders
}

Trabalho realizado pelo grupo de pesquisa em vitamina A do Instituto de Nutrição Josué de Castro da Universidade Federal do Rio de Janeiro, Rio de Janeiro, RJ

\author{
*Correspondência: \\ Universidade Federal do Rio \\ de Janeiro \\ Departamento de Nutrição \\ Social e Aplicada \\ Av. Brigadeiro \\ Tromporwsky, s/n ${ }^{\circ}$ \\ Centro de Ciências da \\ Saúde, bloco $\mathrm{J}, 2^{\circ}$ andar \\ Cep: 21941.590 \\ Rio de Janeiro, RJ \\ Tel/Fax: (21) 2280-8343 \\ Tel: (21) 2560-8293 \\ E-mail: \\ aramalho@rionet.com.br
}

\begin{abstract}
RESUMO
OBjEtivo. Investigou-se a associação entre deficiência de vitamina A (DVA) e condições sociodemográficas em 291 puérperas de diferentes estratos socioeconômicos e seus respectivos recém-nascidos (RN), atendidos em uma maternidade pública do municíio do Rio de Janeiro, Brasil.

Métodos. Os níveis séricos de retinol materno e no sangue de cordão umbilical foram determinados segundo o método Bessey et al. modificado.

Resultados. As prevalências de DVA (retinol sérico <1,05 umol/L) nas puérperas e RN foram de 22\% e 54,2\%, respectivamente. A DVA materna apresentou-se fortemente associada com a DVA nos RN $(p<0,0001)$. A DVA materna não apresentou associação estatisticamente significativa com as variáveis sociodemográficas - escolaridade materna e renda familiar per capita. Tal achado leva a pensar que mesmo em populações nas quais a DVA subclínica seja a forma mais prevalente, o concepto também tem risco aumentado de nascer deficiente.

Conclusã̃o. A carência em questão é um problema significativo entre as puérperas e seus RN e a sua investigação deve ser incorporada às rotinas de assistência pré-natal. Modificação das práticas e da qualidade da dieta como estratégias de combate à DVA, dirigidas aos grupos populacionais vulneráveis, devem dedicar especial atenção às gestantes, independentemente do nível sociodemográfico.
\end{abstract}

UntieRmos: Deficiência de vitamina A. Gestação. Recém-nascido. Puérperas. Condições sociodemográficas.

\section{INTRODUÇÃo}

Segundo estimativa mundial do grupo canadense The Micronutrient Iniciative! , cerca de dois bilhões de pessoas sofrem as conseqüências da fome oculta, na qual se incluem as deficiências de vitamina A (DVA), ferro e iodo.

Estudos realizados na Ásia, a partir de 1995, são unânimes em apontar a maior vulnerabilidade de gestantes e nutrizes portadoras de DVA para o desenvolvimento de intercorrências gestacionais e processos infecciosos mais severos, bem como a maior desvantagem para seus filhos. Essa desvantagem é expressa em maiores taxas de mortalidade materna e dos lactentes nos primeiros seis meses de vida ${ }^{2-6}$.

A Organização Pan-Americana da Saúde ${ }^{7}$ declara que a DVA ainda é considerada um problema de saúde pública em países em desenvolvimento. Além de ser a causa mais importante de cegueira entre as crianças, também contribui significativamente para 0 aumento das taxas de morbi-mortalidade associadas aos processos infecciosos comuns na infância. A DVA na região das Américas prevalece como uma enfermidade subclínica generalizada em muitos países, inclusive no Brasil.

Alguns aspectos relacionados com a DVA no binômio mãe-filho são apontados na literatura. Os ajustes fisiológicos da gestação, a alteração da proporção das proteínas circulantes de vitamina A holo e apo RBP - retinol binding protein, refletem modificações no transporte de retinol durante a gestação $0^{3,8}$. Além disso, a dieta habitual pré-gestacional insuficiente em vitamina $\mathrm{A}$ pode ocasionar baixa reserva hepática do nutriente, que, se associada à persistente ingestão dietética deficiente da vitamina durante o período gestacional, particularmente no terceiro trimestre 9 , aumenta o risco para o desenvolvimento da DVA e da cegueira noturna $(\mathrm{XN})$ gestacional.

Altas prevalências de XN são descritas em gestantes e nutrizes ${ }^{10-12}$. Tal sintoma ocular é, na maioria dos casos, de origem nutricional e manifesta-se em situações de menores níveis circulantes de retinol. $\mathrm{O}$ desenvolvimento da $\mathrm{XN}$ gestacional mostrou-se, em alguns estudos, associada com maior risco de infecções, complicações gestacionais, anemia e maior mortalidade materna e dos lactentes ${ }^{3-6}$. Com isso, a presença de XN passa a ser sugerida como marcador de gestação de alto risco ${ }^{10}$. Em estudo recente realizado no Rio de Janeiro, Brasil, prevalência significativa de XN gestacional também foi descrita entre puérperas atendidas em maternidade pública do município do Rio de Janeiro ${ }^{13}$.

Face ao exposto, a identificação precoce dos indivíduos em risco de desenvolver a DVA deve ser uma preocupação do setor 
de saúde, bem como das características sociodemográficas associadas com a deficiência nutricional, na tentativa de direcionamento dos recursos do setor de saúde para as medidas de intervenção voltadas para os grupos mais vulneráveis. Objetivou-se, no presente estudo, avaliar a associação entre o estado nutricional de vitamina $A$ e as condições sociodemográficas de mães e RN, atendidos em maternidade pública do município do Rio de Janeiro, Brasil.

\section{Métodos}

A população estudada foi constituída por puérperas atendidas pelo Serviço de Assistência Pré-natal da Maternidade Escola da Universidade Federal do Rio de Janeiro (UFRJ). A referida unidade de saúde atende gratuitamente em torno de 1400 a 1500 parturientes por ano, provenientes de várias regiões do município do Rio de Janeiro, RJ, região sudeste do Brasil.

A seleção das 29l participantes do estudo ocorreu no momento da sua admissão para o parto, de acordo com a demanda espontânea à maternidade, em dias alternados de quatro plantões semanais, sendo incluídos dia do final de semana e plantão noturno. A coleta de dados ocorreu após a aprovação do estudo pela comissão de ética da referida unidade.

Todas as mulheres internadas para realização do parto nos dias de coleta de dados que assinaram o termo de consentimento e que atenderam aos critérios de inclusão (gestação de feto único, não portadoras de enfermidades crônicas, não utilização de suplementos vitamínico-minerais contendo vitamina $A$ no período gestacional) foram entrevistadas e realizou-se consulta aos seus respectivos prontuários, visando o preenchimento de questionário pré-testado com informações maternas e dos RN.

As participantes receberam orientação nutricional após a coleta de dados e as diagnosticadas com DVA receberam suplementação de vitamina $A$, segundo recomendação da $O M S^{14}$. Conforme o desenho escolhido para a seleção da amostra e procedimentos analíticos e estatísticos, trata-se de um estudo descritivo do tipo transversal ${ }^{15}$.

Para avaliação do estado nutricional de vitamina $A$, foram coletadas amostras de $5 \mathrm{ml}$ de sangue obtidas por punção venosa das puérperas, bem como de sangue do cordão umbilical dos RN, obtidas por ordenha imediatamente após o parto. Os níveis de retinol sérico foram avaliados segundo o método Bessey et al. modificado $^{16}$ e foram apresentados em classes intervalares de $0,35 \mu \mathrm{mol} / \mathrm{L}$. A DVA foi definida como níveis séricos de retinol inferiores a $1,05 \mu \mathrm{mol} / \mathrm{I}^{3}$.

A renda familiar per capita foi expressa em salários mínimos (SM) e categorizada em quatro grupos: < I SM, I a 2 SM, 3 a 4 SM e $\geq 5$ SM. O grau de escolaridade das puérperas foi classificado em: analfabeta; ensino fundamental incompleto; ensino fundamental completo; ensino médio incompleto; ensino médio completo; e ensino superior. As condições de saneamento foram consideradas adequadas, quando estavam disponíveis os serviços de água ligada à rede pública com canalização interna, esgoto ligado à rede pública e coleta regular de lixo; e inadequadas quando um dos serviços de saneamento não estava disponível.

Considerou-se como participação em programa de assistência pré-natal a paciente que realizou, no mínimo, uma consulta pré-natal por trimestre gestacional.

Visando garantir a qualidade das informações obtidas, os pesquisadores foram treinados e reciclados periodicamente e orientados a seguir o manual de instruções elaborado para a padronização dos procedimentos.

$\mathrm{Na}$ análise dos dados, realizou-se uma análise exploratória visando a identificação dos outliers. Na análise bivariada, verificou-se a associação entre as variáveis categorizadas por meio do teste Qui-quadrado. O nível de significância estabelecido para os testes estatísticos foi $p<0,05$. As análises foram realizadas no pacote estatístico SPSS versão 10 .

\section{Resultados}

Das 29I puérperas estudadas, a média de idade observada foi de 24,5 anos (desvio padrão - DP \pm 6,39), sendo que 67,1\% tinham entre 20 e 35 anos, 60\% com renda familiar per capita maior ou igual a 3 SM e $65,7 \%$ apresentavam o ensino fundamental completo. A maioria teve acompanhamento pré-natal (96,2\%), com uma freqüência média de cinco consultas. Quanto aos RN, 56,2\% eram do sexo masculino, o peso médio observado foi de 3.139 gramas (DP $\pm 0,533$ ) e a idade gestacional ao nascer média foi de 38,6 semanas (DP $\pm 2,43$ ).

A caracterização sociodemográfica das puérperas e $\mathrm{RN}$, segundo o estado nutricional de vitamina A, pode ser encontrada na Tabela I e 2. Não foi evidenciada associação significativa entre o estado nutricional de vitamina $A$ do binômio mãe-filho com as variáveis idade materna, grau de escolaridade materna e renda familiar per capita.

A prevalência de DVA materna foi de $22 \%$, sendo a concentração média de retinol sérico de I,60 $\mu \mathrm{mol} / \mathrm{L}(\mathrm{DP} \pm$ 0,67). Entre os RN, a média dos níveis de retinol no sangue de cordão foi 0,98 $\mathrm{mmol} / \mathrm{L}(\mathrm{DP} \pm 0,45)$, sendo a prevalência de DVA de $54,2 \%$, com um total de II,2\% de valores abaixo de 0,35 $\mu \mathrm{mol} / \mathrm{L}$ (Tabela 3). Foi observado, ainda, que os valores médios dos níveis de retinol no sangue de cordão corresponderam a 61 , 1\% da concentração média de retinol no sangue materno.

Foi encontrada associação significativa entre os níveis de retinol sérico materno e dos RN ( $p<0,000$ I; Tabela 4). O valor médio de retinol no sangue de cordão foi significativamente menor $(0,78 \pm$ 0,50 $\mu \mathrm{mol} / \mathrm{L}$ ) em filhos de mães com DVA comparado com o encontrado entre filhos de mães com adequado estado nutricional - nível de retinol sérico adequado - $(\mathrm{I}, 04 \pm 0,42 \mu \mathrm{mol} / \mathrm{L} ; \mathrm{p}<0,000 \mathrm{I})$. 
Tabela I - Níveis de retinol sérico de puérperas, segundo características sociodemográficas. Maternidade Escola/UFRJ, Rio de Janeiro (I999-2000)

\begin{tabular}{|c|c|c|c|c|c|c|}
\hline \multirow[t]{3}{*}{ Características } & \multicolumn{4}{|c|}{ Níveis de retinol sérico } & \multirow[t]{3}{*}{ Total } & \multirow[t]{3}{*}{$\chi^{2}(p)$} \\
\hline & \multicolumn{2}{|c|}{ DVA* $^{*}$} & \multicolumn{2}{|c|}{ Adequado** } & & \\
\hline & $\mathrm{n}$ & $\%$ & $\mathrm{n}$ & $\%$ & & \\
\hline \multicolumn{7}{|l|}{ Idade materna (anos) } \\
\hline$<20$ & 19 & 26.8 & 57 & 73.2 & 71 & $2.02(0.37)$ \\
\hline 20 a 35 & 37 & 19.1 & 157 & 80.9 & 194 & \\
\hline$\geq 35$ & 6 & 25.0 & 18 & 75.0 & 24 & \\
\hline \multicolumn{7}{|c|}{ Renda familiar per capita (salários mínimos) } \\
\hline$<1$ & 0 & 0 & 5 & 100.0 & 5 & $1.14(0.77)$ \\
\hline $1 \mathrm{a} 2$ & $\|$ & 17.7 & 51 & 82.3 & 62 & \\
\hline 3 a 4 & 8 & 18.6 & 35 & 81.4 & 43 & \\
\hline$\geq 5$ & 9 & 16.4 & 46 & 83.6 & 55 & \\
\hline \multicolumn{7}{|l|}{ Grau de escolaridade } \\
\hline Analfabeta & | & 16.7 & 5 & 83.3 & 6 & $3.53(0.62)$ \\
\hline Ensino fundamental incompleto & 15 & 22.7 & 51 & 77.3 & 66 & \\
\hline Ensino fundamental completo & 14 & 20.6 & 54 & 79.4 & 68 & \\
\hline Ensino médio incompleto & 2 & 8.7 & 21 & 91.3 & 23 & \\
\hline Ensino médio completo & 5 & 12.5 & 35 & 87.5 & 40 & \\
\hline Superior & I & 14.3 & 6 & 85.7 & 7 & \\
\hline
\end{tabular}

* $<1,05 \mu \mathrm{mol} / \mathrm{L} ;$ *** $\geq 1,05 \mu \mathrm{mol} / \mathrm{L}$

Tabela 2 - Níveis séricos de retinol dos recém-nascidos segundo as características sociodemográficas maternas.

Maternidade Escola/UFRJ, Rio de Janeiro (1999-2000)

\begin{tabular}{|c|c|c|c|c|c|c|}
\hline \multirow[t]{3}{*}{ Características } & \multicolumn{4}{|c|}{ Níveis de retinol sérico } & \multirow[t]{3}{*}{ Total } & \multirow[t]{3}{*}{$\chi^{2}(\mathrm{p})$} \\
\hline & \multicolumn{2}{|c|}{ DVA* } & \multicolumn{2}{|c|}{ Adequado** } & & \\
\hline & $\bar{n}$ & $\%$ & $\mathrm{n}$ & $\%$ & & \\
\hline \multicolumn{7}{|l|}{ Idade materna (anos) } \\
\hline$<20$ & 42 & 59.2 & 29 & 40.8 & 71 & $3.51(0.17)$ \\
\hline 20 a 35 & 103 & 53.9 & 88 & 46.1 & $|9|$ & \\
\hline$\geq 35$ & 8 & 36.4 & 14 & 63.6 & 22 & \\
\hline \multicolumn{7}{|c|}{ Renda familiar per capita (salários mínimos) } \\
\hline$<1$ & । & 20.0 & 4 & 80.0 & 5 & $4.61(0.20)$ \\
\hline $1 \mathrm{a} 2$ & 35 & 57.4 & 26 & 42.6 & 61 & \\
\hline 3 a 4 & 18 & 42.9 & 24 & 57.1 & 42 & \\
\hline$\geq 5$ & 30 & 56.6 & 23 & 43.4 & 53 & \\
\hline \multicolumn{7}{|l|}{ Grau de escolaridade } \\
\hline Analfabeta & | & 16.7 & 5 & 83.3 & 6 & $9.44(0.09)$ \\
\hline Ens. fundamental incompleto & 41 & 63.1 & 24 & 36.9 & 65 & \\
\hline Ens. fundamental completo & 37 & 55.2 & 30 & 44.8 & 67 & \\
\hline Ens. médio incompleto & 9 & 39.1 & 14 & 60.9 & 23 & \\
\hline Ens. médio completo & 24 & 64.9 & 13 & 35.1 & 37 & \\
\hline Superior & 3 & 42.9 & 4 & 57.1 & 7 & \\
\hline
\end{tabular}

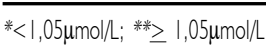

\section{Discussão}

No presente estudo, foi encontrada prevalência significativa de DVA em puérperas e seus RN atendidos em maternidade pública do município do Rio de Janeiro. Tais resultados confirmam a importância da DVA no município, conforme outros achados descritos pelo Grupo de Pesquisa em Vitamina A (GPVA) do Instituto de Nutrição Josué de Castro da Universidade Federal do Rio de Janeiro, em gestantes, puérperas e recém-nascidos atendidos em serviços públicos de saúde do Rio de Janeiro, segundo os indicadores dietético e bioquímico ${ }^{17-21}$.

É reconhecido que a ingestão de vitamina $A$ e as concentrações de retinol sérico durante a gestação influenciam a composição do leite 


\begin{tabular}{|c|c|c|c|c|}
\hline Níveis de retinol ( $\mu \mathrm{mol} / \mathrm{L})$ & & do nu & vitan & \\
\hline & & & $\operatorname{Rec}$ & ascido \\
\hline & $\mathrm{n}$ & $\%$ & $\mathrm{n}$ & $\%$ \\
\hline$<0,35$ & 7 & 2,4 & 32 & 11,2 \\
\hline $0,35 \mid-0,70$ & 17 & 5,8 & 48 & 16,8 \\
\hline $0,70 \mid-1,05$ & 40 & 13,7 & 75 & 26,2 \\
\hline$\geq 1,05$ & 227 & 78,0 & $|3|$ & 45,8 \\
\hline Total & 291 & 100 & 286 & 100 \\
\hline
\end{tabular}

\begin{tabular}{|c|c|c|c|c|c|c|}
\hline \multirow[t]{3}{*}{$\begin{array}{l}\text { Níveis séricos de } \\
\text { retinol das puérperas }\end{array}$} & \multicolumn{4}{|c|}{$\begin{array}{l}\text { Níveis séricos de retinol } \\
\text { dos recém-nascidos }\end{array}$} & \multirow[t]{2}{*}{ Total } & \multirow[t]{2}{*}{$\chi^{2}(p)$} \\
\hline & \multicolumn{2}{|c|}{ DVA* } & \multicolumn{2}{|c|}{ Adequado** } & & \\
\hline & $\begin{array}{l}n \\
51\end{array}$ & $\begin{array}{c}\% \\
79.7\end{array}$ & $\begin{array}{l}n \\
13\end{array}$ & $\begin{array}{c}\% \\
20.3\end{array}$ & 64 & $\begin{array}{r}21,58 \\
(<0,0001)\end{array}$ \\
\hline dequado" & 104 & 46.8 & 118 & 53.2 & 222 & \\
\hline
\end{tabular}

${ }^{*}<1,05 \mu \mathrm{mol} / \mathrm{L} ;{ }^{*} \geq 1,05 \mu \mathrm{mol} / \mathrm{L}$

materno ${ }^{22}$. Assim, um aporte satisfatório de vitamina $A$, na gestação e no período de lactação, pode garantir suprimento adequado ao lactente nos primeiros meses de vida. A freqüência de DVA no binômio mãe-filho encontrada no presente estudo chama a atenção para um quadro preocupante, sobretudo pela necessidade de reposição das quantidades transferidas diariamente ao leite materno, considerado, quantitativamente, a mais importante fonte de vitamina A para o RN já que, normalmente, 60 vezes mais vitamina A é transferida da mãe para o filho durante seis meses de lactação comparada à acumulação feita pelo feto durante os nove meses de gestação 23 .

Cerca de $54,2 \%$ dos RN apresentaram níveis séricos de retinol abaixo do ponto de coorte de I, $05 \mu \mathrm{mol} / \mathrm{L}$, o que sugere que estes RN podem desenvolver DVA nos primeiros anos de vida. Em geral, os níveis de vitamina A no sangue de cordão são inferiores aos maternos, sendo a proporção da concentração plasmática de vitamina $A$ do sangue materno em relação ao sangue fetal de, aproximadamente, 2: I . A baixa concentração de vitamina A sérica ao nascer deve-se à barreira natural, e necessária, imposta pela placenta ${ }^{24}$. Entretanto, durante a gestação, vários fatores podem afetar o estado nutricional materno de vitamina $A$ $\mathrm{e}$, conseqüentemente, a biodisponibilidade para o feto, dentre os quais: a baixa reserva hepática materna; redução da síntese da RBP, o que traz prejúzos ao transporte da vitamina A sérica; deficiência de zinco e o aumento da utilização da vitamina A corporal em processos infecciosos durante a gestação $0^{25-27}$.

O presente estudo evidenciou uma associação entre o estado nutricional de vitamina A materno e níveis de retinol no sangue de cordão umbilical de RN, além de demonstrar frequêencia de valores de retinol abaixo de I,05 $\mu \mathrm{mol} / \mathrm{L}$ no sangue de cordão umbilical maior em mães que apresentavam DVA. Assim, supõe-se que a gestação e a lactação sejam momentos biológicos que mereçam o máximo de atenção em termos de tratamento e prevenção desta carência nutricional, visto que $O$ atendimento às necessidades nutricionais de vitamina $A$ fetais e da criança poderia reduzir a prevalência de DVA, garantindo crescimento e desenvolvimento saudáveis e maior proteção contra as infecções de impacto sobre a saúde e sobrevivência infantis.

A epidemiologia da DVA tem sido geralmente bem estudada em crianças de idade pré-escolar, não apenas em seus aspectos biológicos como também em seus fatores determinantes ${ }^{27-29}$. Com exceção das situações de extrema pobreza, a renda e escolaridade parecem não ter relação na determinação desta condição carencial, reforçando a tese de que a ingestão inadequada de alimentos fonte de vitamina A seja o principal fator etiológico da carência desta vitamina, e que sua exclusão ou baixo consumo estão mais relacionados a questões culturais e hábitos alimentares do que a fatores econômicos $18,28,30-31$. Segundo Van Schaik ${ }^{32}$, grupos biológicos com nível socioeconômico extremamente baixo passam por severa privação nutricional, mas logo acima desta condição de extrema pobreza os recursos gastos na aquisição de alimentos não estão relacionados com a qualidade nutricional da dieta.

Objetivando determinar a prevalência de DVA em pré-escolares e analisar alguns de seus fatores determinantes no semi-árido da Bahia, Santos et al..$^{33}$ demonstraram que $69 \%$ da população estudada apresentava valores de retinol sérico abaixo de 1,05 $\mu \mathrm{mol} / \mathrm{L}$. Entretanto, a DVA não mostrou associação com a escolaridade materna nem com renda familiar per capita. Foi observada, portanto, a mesma prevalência de deficiência de retinol sérico nos estratos mais baixos da população comparada às outras faixas de renda estudadas. As condições socioeconômicas também não apresentaram influência significativa na qualidade da dieta ingerida por crianças americanas entre I a 10 anos de idade em estudo desenvolvido por Johnson et al..$^{34}$. A pesquisa revelou, ainda, ingestão dietética inadequada para diversos micronutrientes, incluindo a vitamina $A$, em diferentes estratos socioeconômicos.

Coelho et al. ${ }^{18}$ avaliaram o estado nutricional de vitamina $A$ de gestantes atendidas em maternidade pública do Rio de Janeiro, tendo descrito inadequação dietética em $12,4 \%$ das entrevistadas e não tendo encontrado associação significativa entre o consumo de vitamina A e as variáveis sociodemográficas (renda familiar per capita e grau de escolaridade).

Accioly \& Souza-Queiróz ${ }^{17}$ encontraram, entre gestantes no terceiro trimestre, acompanhadas em serviço pré-natal de maternidade pública no município do Rio de Janeiro, cifras de 12,5\% de DVA, segundo níveis de retinol sérico, e inadequação dietética da ordem de 14,8\%, segundo método de freqüência de consumo alimentar semiquantitativa. Nesse mesmo estudo, foi verificada a associação entre ingestão e níveis séricos de vitamina $A$, porém não houve associação com a condição socioeconomicodemográfica da clientela, com base nas variáveis renda familiar per capita, escolaridade e idade materna, saneamento e índice de aglomeração da residência. 
Dados do presente estudo corroboram os achados acima relatados que apontam a falta de associação entre a DVA e condição sociodemográfica. Tal constatação aponta para o baixo consumo deste nutriente como fator determinante da carência e o aumento da ingestão de alimentos fonte como uma importante estratégia, no combate à carência no grupo materno-infantil. Neste particular, cabe reflexão sobre a prática da educação nutricional no país, com vistas ao desenvolvimento de técnicas que conduzam à modificações de hábitos alimentares, principalmente dirigidas aos grupos de risco nutricional.

O grupo chave para implementação de modificações de práticas dietéticas no meio familiar e na comunidade representa as mulheres, principalmente as que se encontram em idade reprodutiva, visto que, geralmente, são elas que controlam as práticas alimentares da família, principalmente nos períodos de aleitamento natural, alimentação complementar, desmame e alimentação das crianças em idade pré-escolar ${ }^{35}$.

A prática de transferência de informações aos formadores de opinião como profissionais envolvidos com veículos de comunicação de massa tem-se mostrado um excelente canal para mudanças desejáveis no comportamento da população, devendo, portanto, serem acionados mecanismos que promovam tal articulação. Essas ações seriam reforçadas com programas de educação em saúde e nutrição que contemplem informações a partir da pré-escola até o ensino médio, e com maior aprofundamento das questões nutricionais em nível universitário, visando a reestruturação de hábitos alimentares com vistas ao controle de carências nutricionais.

\section{Conclusão}

Os resultados do presente estudo apontam uma alta prevalência de níveis inadequados de retinol em puérperas e no sangue de cordão umbilical. A prevalência de DVA nos RN foi comparável às cifras encontradas nas regiões mais pobres do mundo e sugerem a necessidade de especial atenção a este grupo populacional por destacar-se dentre os grupos de risco como o mais vulnerável aos efeitos deletérios da carência marginal de vitamina $A$, devido ao rápido crescimento nos primeiros meses de vida.

A falta de associação significativa entre níveis de retinol sérico e a condição sociodemográfica revelada no presente estudo reforça a tese de que a ingestão inadequada de alimentos fonte de vitamina A seja o principal fator etiológico da carência desta vitamina. Entretanto, o aumento da ingestão total de alimentos não necessariamente aumentará o consumo de alimentos fonte de vitamina $A$, uma vez que a seleção de alimentos é muito complexa e influenciada por diversos outros fatores, além do acesso aos alimentos e o conhecimento de nutrição.

O profissional de saúde, ao sugerir qualquer intervenção na área alimentar com vistas ao combate da DVA, deve ponderar os aspectos não só econômicos mas também os culturais relacionados, principalmente quando a proposta de intervenção envolva aspectos educativos. Medidas que concorram para a modificação das práticas alimentares e da qualidade da dieta como estratégia de combate à carência de vitamina $A$, em nível populacional, com base no incremento da ingestão de alimentos fonte e fortificados com vitamina $A$, devem ser incluídas no elenco de ações universais da atenção pré-natal, independentemente das condições socioeconômicas da clientela, o que pode contribuir para a melhoria do estado nutricional de vitamina A materno e dos RN.

\section{Conflito de interesse: não há}

\section{SUMmaRY}

Association betWeEn maternal and NeWBorn Vitamin a STATUS AND ECONOMIC STRATUM IN RIO DE JANEIRO, BRAZIL

BACKGROUND. Retinol was determined spectrophotometrically in the blood of 29 I mothers at delivery and in the umbilical cord of the newborn. The mothers came from different socioeconomic strata in Rio de Janeiro.

METHODS. Levels of retino/ were determined using spectrophotometry (Bessey et al).

RESULTS. The circulationlevels of retinolwerelow (below $1.05 \mu \mathrm{mo} / \mathrm{L}$ ) in $22.0 \%$ of the mothers and in $54.2 \%$ of the newborn. A close association was found between low levels in the mothers' serum and those in the children's' umbilical cord $(p<0.000$ I). There was no association between maternal vitamin A deficiency (DVA) and socioeconomic stratum, family income or level ofeducation.

CONCLUSIONS. DVAis a distressing health problemamongmothers and newborns and its diagnosis must be included in antenatal care. This reinforces the concept that intervention programs must emphasize changes in feeding practices and focus on the most vulnerable groups, of which pregnant women warrant special attention, regardless of socioeconomic stratum. [Rev Assoc med Bras 2006; 52(3): 170-5]

KEY WORDS: Vitamin A deficiency. Pregnancy. Lactating women. Newborn. Socioeconomic stratum.

\section{REFERÊNCIAS}

I. Mason JB Lotfi M, Dalmiya N, Sethuraman K, Deitchler M. The Micronutrient Report. Current progress and trends in the control of vitamin A, iodine, and iron deficiencies. Ottawa: The Micronutrient Iniciative/UNICEF; 200 I.

2. Biswas AB, Mitra NK, Chakraborty I, Basu S, Kumar S. Evaluation of vitamin A status during pregnancy. Indian Med Assoc 2000;98:525-9.

3. Christian P, West JR KP, Khatry SK, Katz J, Shrestha SR, KimbroughPradhan E, et al. Night blindness of pregnancy in rural Nepal: nutritional and health risks. Int J Epidemiol 1998;27:23 I-7.

4. Christian P, West JR Kp, Khatry Sk, Kimbrough-Pradhan E, Leclerq, S.C.; Katz, J et al. Night blindness during pregnancy and subsequent mortality among women in Nepal: Effects of vitamin A and ?-carotene supplementation. Am J Epidemiol 2000, I 52:542-7.

5. Christian P, West KP Jr, Khatry SK, Katz J, Leclerq SC, Kimbrough-Pradhan $E$, et al. Maternal night blindness increases risk of mortality in the first 6 months of life among infants in Nepal. J Nutr 200 I | 3 | : I 5 I0-2.

6. Radhika MS, Bhaskaram P, Balakrishna N, Ramalakshmi BA, Devi S, Kumar BSs. Effects of vitamin A deficiency during pregnancy on maternal and child health. BJOG: Int J Gynaecol Obstet 2002; I 09:689-93.

7. Organización Panamericana de la Salud. Visión Integrada de la suplementación con vitamina A en las Américas. 2-4 de mayo del 200 I, Managua, Nicaragua. Informe de la Reunión Regional; 2001 . (HPP/HPN/ MN/49-17). Washington: OPS; 2001.

8. Sapin V, Alexandre MC, Chaïb S, Bournazeau JA, Sauvant P, Borel P, et al. Effect of vitamin A status at the end of term pregnancy on the saturation of retinol binding protein with retinol. Am J Clin Nutr 2000; 71:537-43. 
9. Institute of Medicine VitaminA. In: Dietary reference intakes for vitamin A vitamin K, arsenic, boron, chromium, cooper, iodine, iron, manganese, molybdenum, nickel, silicon, vanadium, and zinc. Washington: National Academy Press; 2001. p.82-161.

10. Christian P. Recommendations for indicators: night blindness during pregnancy - a simple tool to assess vitamin A deficiency in a population. J Nutr 2002, 132:2884S-8S.

I I. International Vitamin A Consultative Group. IVACG Statement. Materna Night Blindness: a new indicator of vitamin A deficiency. Washington: IVACG; 2002.

12. Katz J, Khatry SK, West KP Jr, Humphrey JH, Leclerq SC, Pradhan EK, et al. Night blindness is prevalent during pregnancy and lactation in rural Nepal. J Nutr 1995; | 25:21 22-7.

13. Saunders C, Leal MC, Gomes MM, Campos LFC, Dos Santos Silva BA, Lima APPT, et al. Gestational nightblindness in women treated at a public maternity hospital in Rio de Janeiro, Brazil. J Health Popul Nutr 2004;22:348-56

14. Organisation Mondiale de la Santé. Supplémentation en vitamine A. Genève: OMS; 1998

15. Hennekens $\mathrm{CH}$, Buring JE. Epidemiology in medicine. Boston: Little, Brown and Company; 1987.

16. Araújo CRC, Flores H. Improved spectrophotometric vitamin A assay Clin Chem 1978;24:386.

17. Accioly E, Souza-Queiróz S. Deficiencia de vitamina A en embarazadas asistidas en una maternidade publica em Rio de Janeiro, Brasil. Rev Chil Nutr 2001;27:352-7.

18. Coelho CSP, Ramalho RA, Accioly E. O inquérito dietético na avaliação do estado nutricional de vitamina A em gestantes. Ars Curandi Clin Med 1995:6:44-60.

19. Ramalho RA, Anjos LA, Flores $H$. Estado nutricional de vitamina A no binômio mãe/recém-nascido em duas maternidades no Rio de Janeiro, Brasil. Arch Latinoam Nutr 1999;49:3 18-2 I

20. Ramalho RA, Saunders C, Paiva F, Accioly E, Cardoso LO, Natalizi D. Estado de vitamina $A$ de puérperas e recém-nascidos e estado antropométrico materno. Rev Cienc Med PUCCAMP 200 I ; 0:5- 10.

21. Ramalho RA, Flores H, Saunders C. Hipovitaminose A: Um Problema de Saúde Pública no Brasil. Hypovitaminosis A: a public health problem in Brazil. Rev Panam Salud Pública 2002; I2: I 17-22.

22. Ortega RM, Andres P, Martinez RM, Lopez-Sobaler AM. Vitamin A status during the third trimester of pregnancy in Spanish women: Influence on concentrations of vitamin A in breast milk. Am J Clin Nutr 1997;66:564-8.
23. Stoltzfus RJ, Underwood BA. Breastmilk vitamin A as na indicator to assess vitamin A status of womem and infants. Bull World Health Organ 1995 73:703-II

24. Wallingford JC, Underwood BA. Vitamin A deficiency in pregnancy, lactation, and the nursing child. In: Vitamin A deficiency and its control. Bauernfeind JC, editor. New York: Academic Press; 1986. p. I0I-52.

25. Arroyave G. Interrelations between protein and vitamin $A$ and metabolism. Am J Clin Nutr 1969; 22: I I 19-28.

26. Arroyave $G$, Calcaño $M$. Descenso de los niveles sericos de retinol y su proteina de enlace (RBP) durante las infecciones. Arch Latinoam Nutr 1979; 29:233-60.

27. Sommer A. Vitamin A deficiency and its consequences: A field guide to detection and control: epidemiology. 3rd ed. Geneva: World Health Organization; 1995

28. McLaren DS, Frigg M. Manual de ver y vivir sobre los transtornos por deficiencia de vitamina A (VADD). Washington: OPS; 1999

29. World Health Organization. Indicators for assessing vitamin A deficiency and their application in monitoring and evaluating intervention programmes. WHO/NUT/96.10. Geneva: WHO; 1996

30. Brunken GS, Flores $H$. Why do diets lack vitamin A. Nutriview 1994;3: I-3

31. Ramalho RA, Saunders C. Reflexões sobre alimentação na busca de superar carências nutricionais. Rev Nutr 2000; | 3: | | -6.

32. Van Schaik TF. Food and nutrition relative to family life. J Home Econ 1964;56:225-32.

33. Santos LMP, Assis AMO, Martins MC, Araújo MPN, Morris SS, Barreto MI. Situação nutricional e alimentar de pré-escolares no semi-árido da Bahia (Brazil): II - vitamin A deficiency. Rev Saude Publica 1996;30:67-74.

34. Johnson RK, Guthrie H, Smiciklas-Wright H, Wang MQ. Characterizing nutrient intakes of children by sociodemographic factors. Public Health Rep 1994; 109(3):4 14-20.

35. Underwood BA. Estrategias a largo plazo para el control de las deficiencias de micronutrientes. Vitamin A Field Support Project (VITAL), Arlington, Virginia: USAID; 1993. p.70-76.

Artigo recebido: 06/12/2004 Aceito para publicação: 28/02/2005 